\title{
Resting State Functional Connectivity of the Rat Claustrum
}

\author{
Samuel R. Krimmel ${ }^{1}$, Houman Qadir ${ }^{2}$, Natalie Hesselgrave ${ }^{3}$, Michael G. White ${ }^{2}$, \\ David H. Reser ${ }^{4,5}$, Brian N. Mathur ${ }^{2 * \dagger}$ and David A. Seminowicz ${ }^{1 * \dagger}$
}

${ }^{1}$ Center to Advance Chronic Pain Research, Department of Neural and Pain Sciences, School of Dentistry, University of Maryland, Baltimore, MD, United States, ${ }^{2}$ Department of Pharmacology, School of Medicine, University of Maryland, Baltimore, MD, United States, ${ }^{3}$ Department of Physiology, School of Medicine, University of Maryland, Baltimore, MD, United States, ${ }^{4}$ Graduate Entry Medicine Program, Monash Rural Health-Churchill, Churchill, VIC, Australia, ${ }^{5}$ Department of Physiology, Monash University, Clayton, VIC, Australia

\section{OPEN ACCESS}

Edited by:

Kathleen S. Rockland,

Boston University School of

Medicine, United States

Reviewed by:

lleana Ozana Jelescu,

École Polytechnique Fédérale de

Lausanne, Switzerland

Richard Jarrett Rushmore,

Boston University, United States

${ }^{*}$ Correspondence:

Brian N. Mathur

bmathur@som.umaryland.edu

David A. Seminowicz

dseminowicz@umaryland.edu

${ }^{\dagger}$ Co-senior authors

Received: 08 August 2018 Accepted: 06 February 2019 Published: 22 February 2019

Citation: Krimmel SR, Qadir H, Hesselgrave N, White MG, Reser DH, Mathur BN and Seminowicz DA (2019) Resting State Functional Connectivity of the Rat Claustrum.

Front. Neuroanat. 13:22. doi: 10.3389/fnana.2019.00022
The claustrum is structurally connected with many cortical areas. A major hurdle standing in the way of understanding claustrum function is the difficulty in assessing the global functional connectivity (FC) of this structure. The primary issues lie in the inability to isolate claustrum signal from the adjacent insular cortex (Ins), caudate/putamen (CPu), and endopiriform nucleus (Endo). To address this issue, we used (7T) fMRI in the rat and describe a novel analytic method to study claustrum without signal contamination from the surrounding structures. Using this approach, we acquired claustrum signal distinct from Ins, CPu, and Endo, and used this claustrum signal to determine whole brain resting state functional connectivity (RSFC). Claustrum RSFC was distinct from the adjacent structures and displayed extensive connections with sensory cortices and the cingulate cortex, consistent with known structural connectivity of the claustrum. These results suggest $\mathrm{fMRI}$ and improved analysis can be combined to accurately assay claustrum function.

Keywords: anterior cingulate cortex, caudate, cortex, forebrain, insula, putamen, striatum, top-down

\section{INTRODUCTION}

The claustrum is highly interconnected with many cortical areas (Crick and Koch, 2005; Mathur, 2014). Early work in the cat suggests that the claustrum receives multimodal sensory input (Segundo and Machne, 1956; Spector et al., 1974). This concept was provisionally supported in a human study by Hadjikhani and Roland (1998) who showed that claustrum activation was greatest when both somatosensory and visual modalities were recruited in order to complete an object recognition task. In light of these data and taking the early view of Ettlinger and Wilson (1990) to a new conceptual level, Crick and Koch (2005) presented a new hypothetical framework: claustrum integrates multimodal information for the generation of conscious perception.

Countering the notion that the claustrum binds sensory information, later work in the cat (Olson and Graybiel, 1980; LeVay and Sherk, 1981) and monkey (Remedios et al., 2010) showed that only unimodal responses in the claustrum are detectable. Additionally, the anatomical pattern of claustrum connectivity shows that it weakly innervates primary sensorimotor cortices (White et al., 2017), but heavily innervates the medial prefrontal cortex (White et al., 2017) and anterior cingulate cortex (ACC) in marmoset (Reser et al., 2017) and rodent (Mathur et al., 2009; White et al., 2017, 2018). The ACC also projects heavily to 
claustrum (Smith and Alloway, 2010; White et al., 2017) and provides a top-down preparatory signal that is proportional to cognitive load (White et al., 2018). Moreover, ACC input to mouse claustrum innervates claustrum projection neurons that, in turn, project to parietal association cortex and visual cortices (White and Mathur, 2018). Together these data suggest that the claustrum may support top-down cognitive processing by coordinating multiple, widespread cortical regions. As such, ascertaining the global functional connectivity (FC) of the claustrum is required.

Resting state FC (RSFC) analysis as assessed with fMRI allows us to estimate region-to-whole-brain coupling (McIntosh, 2000). Given that the claustrum is a thin structure interposed between the insula (Ins), caudate/putamen $(\mathrm{CPu})$, and endopiriform nucleus (Endo), it is likely that claustrum signal will have surrounding structures erroneously incorporated into it by virtue of partial effects, in which the signal from voxels within the claustrum also contain signals from tissue outside the claustrum boundaries (González Ballester et al., 2002; Du et al., 2014; Dukart and Bertolino, 2014). The goals of this experiment were to improve methodology used to study small volumes in fMRI and to reveal the RSFC of the claustrum in rats. We used rodent $\mathrm{fMRI}$ with a novel analytic technique called small region confound correction (SRCC) to isolate spontaneous claustrum signal from the Ins, $\mathrm{CPu}$, and Endo to accomplish these goals. SRCC is an easy to implement method where "flanking" sections of structures adjacent to a small region of interest (ROI), like the claustrum, are regressed from the ROI, creating a confoundcorrected ROI timeseries. Using this approach, we find claustrum is functionally connected with ACC, consistent with structural connectivity data (Reser et al., 2017; White et al., 2018) and that claustrum connectivity differs from the neighboring regions. Together these data set the stage for future analysis of claustrum participation in brain networks underlying cognition.

\section{MATERIALS AND METHODS}

\section{Animals}

We used 10 adult female Sprague-Dawley rats (200-250 g, Charles River, DC, USA; 6 weeks) housed in ventilated plastic cages with soft bedding and kept on a 12/12 h light/dark cycle (lights on at 07:00), at constant temperature $\left(22 \pm 2{ }^{\circ} \mathrm{C}\right)$ and humidity $(50 \pm 10 \%)$. Rats consumed standard rat chow and had ad libitum access to water. All procedures were approved by the Institutional Animal Care and Use Committee at the University of Maryland Baltimore and performed in accordance with the National Institutes of Health Guide for the Care and Use of Laboratory Animals.

\section{MRI Acquisition}

MRI data were acquired with a Bruker BioSpec 70/30USR Avance III 7-Tesla scanner (Bruker Biospin MRI GmbH, Ettlingen, Germany) with a BGA12S gradient system and interfaced to a Bruker Paravision 5.1 console. A Bruker $40 \mathrm{~mm}$ circular polarized volume coil was used for data acquisition. During scanning, rats were anesthetized at a constant level of isoflurane $(\leq 1.5 \%)$ and respiration and heart rate were monitored with a small animal monitoring and gating system and software (SA Instruments, Inc., Stony Brook, NY, USA). Anesthesia is well known to modulate the RSFC of the brain (Paasonen et al., 2018). These data were collected as part of a longitudinal study (see Hubbard et al., 2015), and subsequently we did not use terminal anesthetics like $\alpha$-chloralose. Isoflurane may result in less localized brain mapping, so we used a low dose that mitigates these negative effects (Sommers et al., 2009; Williams et al., 2010). However, even low doses of isoflurane produce RSFC maps that differ from the awake brain more than other alternative anesthetics (Paasonen et al., 2018), and isoflurane suppresses network presence relative to medetomidine (Kalthoff et al., 2013). Throughout data acquisition, body temperature was kept at $36-37^{\circ} \mathrm{C}$ using a circulating warm water heater. A T2-weighted image was obtained $(\mathrm{RARE}, \mathrm{TR}=2,000 \mathrm{~ms}, \mathrm{TE}=14 \mathrm{~ms}, 256 \times 256$, in plane resolution $=100 \mu \mathrm{m}, 24$ axial slices, $1 \mathrm{~mm}$ slice thickness) for anatomical reference. Resting state scans were acquired using a spin-echo echo-planar imaging sequence $(\mathrm{TR}=1,500 \mathrm{~ms}, \mathrm{TE}=35.0966 \mathrm{~ms}, 75 \times 75$, in plane

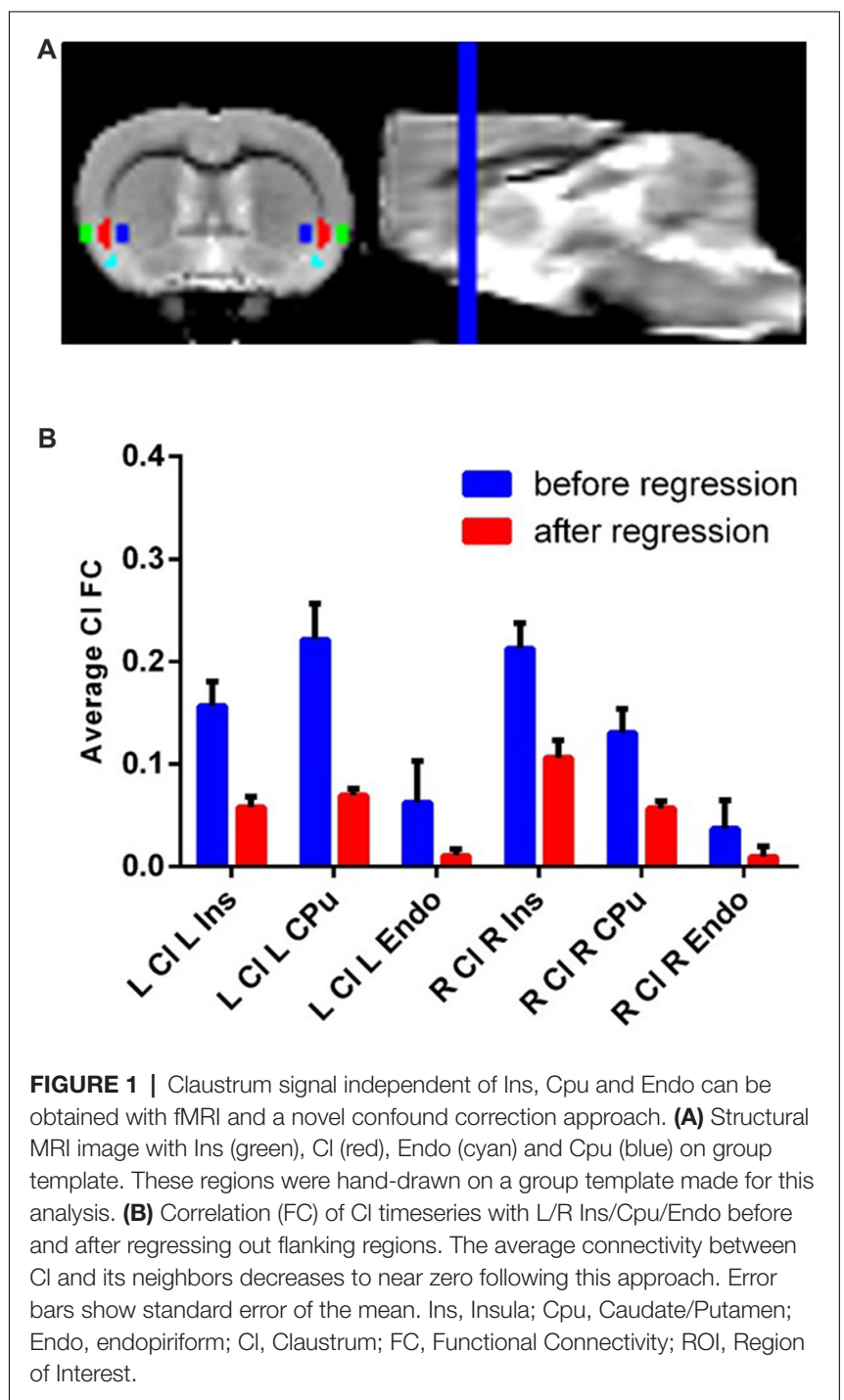


resolution $=0.4 \times 0.4 \mathrm{~mm}, 1 \mathrm{~mm}$ slice thickness, 24 axial slices; 550 volumes).

\section{Preprocessing}

All image preprocessing was conducted using SPM8 ${ }^{1}$. The pipeline included slice timing correction, realignment, normalization, and smoothing with a $1 \mathrm{~mm}$ FWHM Gaussian kernel. We created a study-specific template by coregistering and averaging the T2-weighted images across animals and interpolating to voxel size of isotropic $0.5 \mathrm{~mm}$.

\section{Region of Interest (ROI) Creation}

Each ROI (eight total: bilateral claustrum, Ins, CPu and Endo) was hand drawn on the previously described template brain resliced to $0.3 \mathrm{~mm}$ isotropic voxels (Figure 1A). We identified the claustrum based on known anatomical boundaries of the claustrum and used a parvalbumin staining from a separate dataset as a molecular marker for the claustrum (Qadir et al., 2018). The volume of each (i.e., left and right) claustrum was

${ }^{1}$ http://www.fil.ion.ucl.ac.uk/spm/
$2.86 \mathrm{~mm}^{3}$, or about 18 native resolution voxels. Additionally, we also drew a white matter and cerebro-spinal fluid (CSF) mask on this template.

\section{Small Region Confound Correction (SRCC)}

fMRI suffers from partial volume effects, where voxels for the ROI contain tissues not belonging to the ROI (González Ballester et al., 2002; Du et al., 2014; Dukart and Bertolino, 2014). This creates ROI functional data with varying degrees of contamination from outside structures. This problem is especially pronounced for small structures like the claustrum, because the percentage of voxels with partial volume effects is quite high relative to very large structures. To address this problem, we treated sections of the major surrounding regions of the claustrum, the Ins, $\mathrm{CPu}$, and Endo, as sources of noise, similar to how white matter is typically treated in resting state analysis. Specifically, we dilated the claustrum ROI by two voxels $(0.6 \mathrm{~mm})$ and created an overlap map with the Ins/Cpu/Endo and repeated the process with four dilations as well. Next, we subtracted the 2 nd dilated overlap image from the 4 th dilated

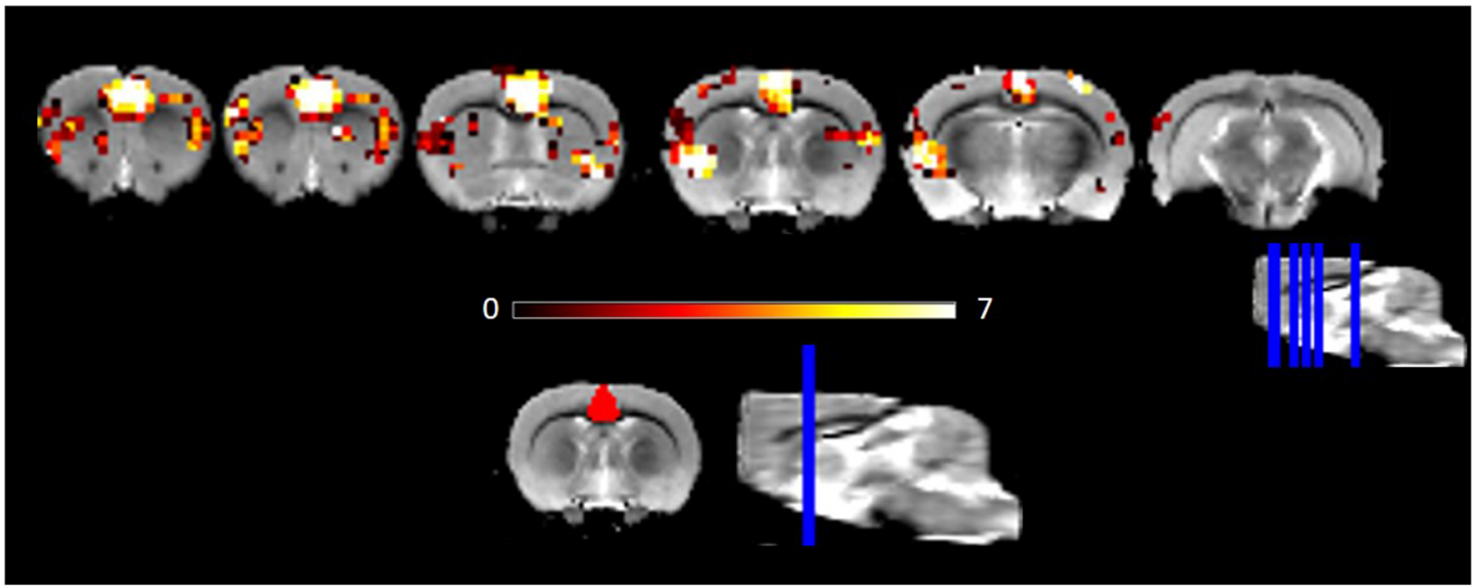

FIGURE 2 | RSFC of the left and right Cl showing connectivity to frontal and posterior cortices. (A) RSFC of the left Cl. (B) RSFC of the right Cl. Data were thresholded at $p<0.001$ followed by FWE cluster correction. Cl, claustrum; RSFC, resting state function connectivity.

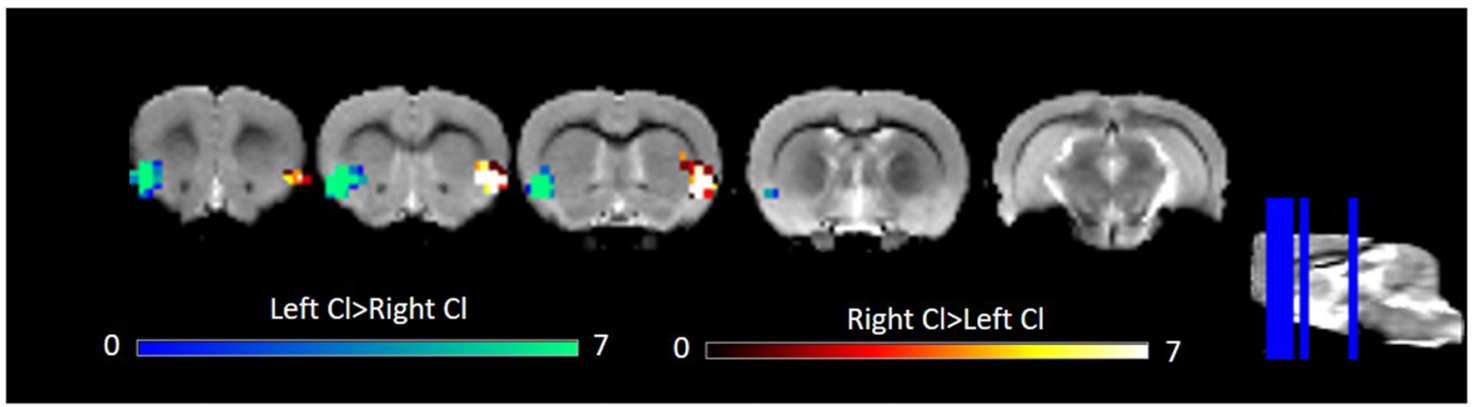

FIGURE 3 | Laterality in RSFC of the left and right Cl. Data were thresholded at $p<0.001$ followed by FWE cluster correction. Cl, claustrum; RSFC, resting state functional connectivity. 
overlap image. This created new "flanking" ROIs out of the medial section of Ins, lateral section of $\mathrm{CPu}$, and dorsal section of Endo that roughly corresponded to the shape of the claustrum. We next treated these as sources of noise in RSFC analysis of the claustrum similar to white matter. This created claustrum FC maps that were linearly independent of partial volume effects from the Ins, $\mathrm{CPu}$ and Endo.

\section{Determining Functional Connectivity}

Resting state preprocessing and seed-based analyses were conducted in the Conn toolbox version $17 \mathrm{f}^{2}$. Given continued controversy, we did not remove global signal from resting state data (Power et al., 2017). To account for noise present

${ }^{2}$ http://www.nitrc.org/projects/conn in white matter and CSF we used aCompCor (Muschelli et al., 2014) to determine the first five eigenvectors of white matter and CSF. We removed motion related signals estimated from the realignment parameters along with the first order derivatives of these parameters, in addition to the 10 previously mentioned eigenvectors for white matter and CSF. To avoid the reintroduction of noise while removing low frequency artifact, we used linear detrending and also simultaneously used a bandpass filter of $0.008-0.1 \mathrm{~Hz}$. Despiking was used after these steps to remove any additional artifact that had yet to be removed (Patel et al., 2014). When determining claustrum connectivity, we also included the "flanking" segments of Ins, $\mathrm{CPu}$, and Endo (see SRCC) as sources of noise. The timeseries for each ROI (excluding CSF and WM) was determined by averaging all voxels within the volume using unsmoothed functional data.

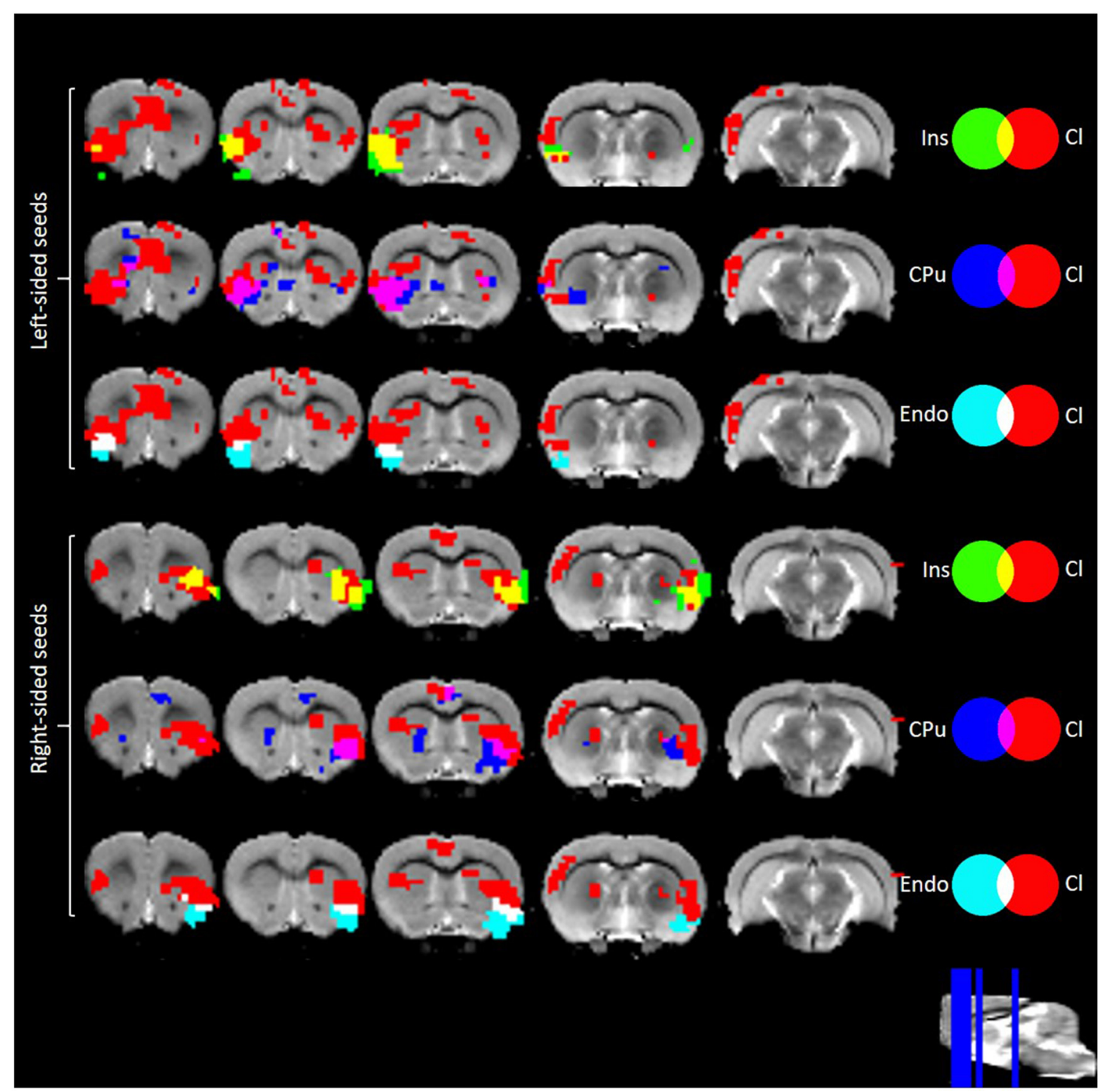

FIGURE 4 | RSFC of Ins, Cpu, Endo, and Cl. These maps indicate a unique pattern of Cl connectivity relative to the surrounding structures. Data were thresholded at $p<0.001$ followed by FWE cluster correction. Cl, claustrum; Ins, Insula; Cpu, Caudate/Putamen; Endo, Endopiriform; RSFC, resting sate functional connectivity. 


\section{Statistics and Data Availability}

To determine significant RSFC, we performed one sample $t$-tests for resting state contrast maps with a cluster-forming threshold of $p<0.001$ and an FWE cluster correction. This threshold has been shown to adequately control for false positive rates (Woo et al., 2014). To determine laterality of claustrum connectivity, paired $t$-tests for left and right claustrum resting state contrast maps with a cluster-forming threshold of $p<0.001$ and an FWE cluster correction were performed. All data including code, ROI images, and the template brain are available upon request.

\section{RESULTS}

\section{Functionally Isolating the Claustrum From Surrounding Structures}

Using resting state data, we observed timeseries correlation for: left claustrum left $\mathrm{CPu} r=0.22$, std $=0.11$; left claustrum left Ins $r=0.16$, std $=0.08$; left claustrum left Endo $r=0.06$, std $=0.13$; right claustrum right $\mathrm{CPu} r=0.13$, std $=0.07$; right claustrum right Ins $r=0.21$, std $=0.08$; right claustrum right Endo $r=0.04$, std $=0.09$. Following SRCC, we observed reduced timeseries correlation for: left claustrum left $\mathrm{CPu} r=0.06$, std $=0.03$; left claustrum left Ins $r=0.07$, std $=0.02$; left claustrum left Endo $r=0.01$, std $=0.02$; right claustrum right $\mathrm{CPu} r=0.06$, std $=0.02$; right claustrum right Ins $r=0.11$, std $=0.05$; right claustrum right Endo $r=0.01$, std $=0.03$ (Figure 1B). Thus, we were able to isolate claustrum signal from the surrounding regions using SRCC. To further validate this approach, we next applied this method to the ACC using bilateral M2 as surrounding regions and found that again, SRCC was able to isolate ROI signal (Supplementary Figure S1).

\section{Functional Connectivity of the Rat Claustrum}

We estimated FC of the corrected claustrum timeseries across the whole brain, anticipating that RSFC would be largely consistent with known anatomical connectivity of the claustrum. We therefore hypothesized that claustrum would have RSFC with sensory cortices and cingulate. Using the right claustrum seed, we observed strong RSFC with the bilateral cingulate, left claustrum, right auditory cortex, posterior area of the parietal cortex, visual cortex, S1, and S2 (Figure 2B). When using the left claustrum seed, we observed RSFC with the cingulate, $\mathrm{CPu}$, auditory cortex, visual cortex, posterior area of the parietal cortex, S1, S2, and entorhinal cortex (Figure 2A).

\section{Laterality of the Rat Claustrum}

Several studies have observed laterality in claustrum activation (Hadjikhani and Roland, 1998; Naghavi et al., 2007; Lerner et al., 2008). Given this previous work and some qualitative differences in left and right claustrum RSFC maps in our data, notably left claustrum RSFC that spanned less of the cingulate than did RSFC of the right claustrum, we sought to quantitatively examine laterality. Using a paired $t$-test, we observed only bilateral clusters containing the claustrum (Figure 3). We did not find evidence for laterality in claustrum RSFC with the cingulate.

\section{Functional Connectivity of Claustrum vs. Surrounding Structures}

Overlaying the RSFC maps of claustrum, Ins, $\mathrm{CPu}$, and Endo bilaterally revealed distinct patterns of connectivity. Left claustrum displayed more extensive cingulate, $\mathrm{S} 1$, and auditory cortex RSFC than did the surrounding regions (Figure 4). The right claustrum displayed more extensive cingulate, S1, auditory cortex, and visual cortex than did the Ins, $\mathrm{CPu}$, or Endo (Figure 4). Notably, the claustrum signal was not treated as a confounding source for the Ins/CPu/Endo. These data further support that we obtained a claustrum signal unique from the surrounding structures and are consistent with the extensive anatomical connectivity of claustrum to cingulate and sensory cortices.

\section{DISCUSSION}

In the current study, we propose a novel approach, SRCC, to study the claustrum using fMRI that overcomes existing problems in the field, namely, the masking of signal from an ROI by incorporating outside tissue into the ROI. This is an especially important problem in claustrum research because small volumes are particularly vulnerable to partial volume effects. Using fMRI and SRCC, we were able to isolate claustrum from Ins, $\mathrm{CPu}$, and Endo, and show that claustrum displays robust RSFC with the cingulate and sensory cortices, consistent with anatomical connectivity.

RSFC is a complex measure that does not precisely mirror structural connectivity. However, RSFC is often consistent with structural connectivity (Greicius et al., 2009) and reflects anatomical features (Gordon et al., 2017). Consistent with a relationship between functional and structural connectivity, our RSFC maps derived from claustrum bear overlap with known anatomical connectivity. The rat claustrum sends dense inputs to the entire cingulate cortex (Wang et al., 2017; White et al., 2017) and the ACC, in particular, sends a dense input to the claustrum (Smith and Alloway, 2010; Wang et al., 2017; White et al., 2017). The claustrum also projects to the auditory and visual cortices (Wang et al., 2017; White et al., 2017; White and Mathur, 2018), the posterior area of the parietal cortex (Wang et al., 2017; White et al., 2017; White and Mathur, 2018), and S1 (Wang et al., 2017; White et al., 2017). Although direct connections between claustrum and the $\mathrm{CPu}$ are not clearly supported in the literature, the claustrum shares connectivity with cortical areas that are structurally connected with the $\mathrm{CPu}$ : the ACC projects to the dorsomedial aspect of the $\mathrm{CPu}$ (Voorn et al., 2004) and visual, auditory, somotasensory and motor cortices project to the dorsolateral aspect of the CPu (McGeorge and Faull, 1989). Taken together, these data support the notion that our claustrum RSFC data are valid.

Using resting state data, we observed minimal similarity between the timeseries of claustrum and $\mathrm{Ins} / \mathrm{CPu} / \mathrm{Endo}$, indicating minor incorporation of the Ins, $\mathrm{CPu}$, and Endo signal into the claustrum ROI. To further isolate claustrum signal we regressed flanking segments of Ins/CPu/Endo from the claustrum in a novel approach called SRCC. SRCC further 
reduced the timeseries similarity between claustrum and the neighboring regions to near zero, meaning that an independent claustrum signal was obtained. There are several limitations with this approach. First, this method assumes that any similarity shared by claustrum and adjacent structures is a partial volume effect, and hence, a confound. Any true FC between claustrum and $\mathrm{Ins} / \mathrm{CPu} /$ Endo was therefore removed in our approach. In rats, there does not appear to be anatomical connectivity between the $\mathrm{CPu}$ and claustrum (Smith and Alloway, 2010), suggesting that $\mathrm{CPu}$ and claustrum should have low RSFC. Some connectivity between Ins and claustrum may exist in in rats (Lipowska et al., 2000; Qadir et al., 2018), which may suggest that some Ins-claustrum FC could have been suppressed with SRCC. Limited RSFC between the claustrum and endopiriform nucleus is also expected given that the endopiriform nucleus and claustrum have distinct anatomical connectivity patterns in mice (Watson et al., 2017; Qadir et al., 2018). An additional limitation of SRCC is regressing out these surrounding signals from claustrum could make an artifactual claustrum signal. However, our resting state analysis showed largely similar bilateral claustrum FC and was consistent with well documented anatomical connectivity, strongly suggesting that the corrected claustrum timeseries is physiological. Following SRCC, we still observe overlap between claustrum RSFC maps and Ins/CPu/Endo RSFC maps. This cannot be a result of partial volume effects from the bordering tissues, because there is no linear relationship between claustrum and Ins/CPu/Endo. These results likely reflect shared functional coupling between these structures and cortical targets. SRCC offers a simple-to-implement methodology that can greatly enhance confidence in small volume studies with fMRI, such as the claustrum, habenula, bed nucleus of the stria terminalis, thalamic association nuclei, and ventral pallidum. Additionally, the value of the approach will increase as the resolution of the fMRI data decreases, potentially making SRCC even more useful in standard resolution human fMRI datasets than in the current dataset.

The choice of our anesthetic is an additional limitation. By virtue of these data being collected as part of a longitudinal design (Hubbard et al., 2015), we were not able to use terminal anesthetics like $\alpha$-chloralose. However, non-terminal anesthetics like metodimine (Nasrallah et al., 2014) have shown more similar

\section{REFERENCES}

Crick, F. C., and Koch, C. (2005). What is the function of the claustrum? Philos. Trans. R. Soc. Lond. B Biol. Sci. 360, 1271-1279. doi: 10.1098/rstb. 2005.1661

Du, Y. P., Chu, R., and Tregellas, J. R. (2014). Enhancing the detection of BOLD signal in fMRI by reducing the partial volume effect. Comput. Math. Methods Med. 2014:973972. doi: 10.1155/2014/973972

Dukart, J., and Bertolino, A. (2014). When structure affects function-the need for partial volume effect correction in functional and resting state magnetic resonance imaging studies. PLoS One 9:e114227. doi: 10.1371/journal.pone. 0114227

Ettlinger, G., and Wilson, W. (1990). Cross-modal performance: behavioural processes, phylogenetic considerations and neural mechanisms. Behav. Brain Res. 40, 169-192. doi: 10.1016/0166-4328(90)90075-p
RSFC to awake animals (Paasonen et al., 2018), and better network structure (Kalthoff et al., 2013) than isoflurane. Future work should experiment with better anesthetic protocols, that will likely result in more reliable and more widespread claustrum RSFC maps.

In conclusion, we show that in rat fMRI combined with SRCC, we can obtain claustrum signal independent of the surrounding structures. Using this claustrum signal, we observed RSFC with strong similarity to known anatomical connectivity of the claustrum. Together these data set the stage for future analysis of claustrum participation in brain networks underlying cognition.

\section{AUTHOR CONTRIBUTIONS}

DS, DR, and BM conceived the research. DS, SK, MW, and BM designed and performed the research. $\mathrm{NH}$ generated claustrum masks. DS, SK, MW, and BM analyzed data. DS, SK, HQ, and $\mathrm{BM}$ wrote the manuscript.

\section{FUNDING}

This work was supported by National Institute on Alcohol Abuse and Alcoholism grants K22AA021414, R01AA024845 (BM), Whitehall Foundation grant 2014-12-68 (BM), National Institute of General Medical Sciences grant T32008181 (MW), National Institute of Neurological Disorders and Stroke grant T32NS063391 (MW), and National Center for Complementary and Integrative Health grant R01AT007176 (DS). DR was supported by NHMRC Project Grant APP1068140.

\section{ACKNOWLEDGMENTS}

The authors also are grateful for the assistance of Dr. Rao Gullapalli and the Core for Translational Research in Imaging @ Maryland (C-TRIM) and the Center for Metabolic Imaging and Therapeutics (CMIT).

\section{SUPPLEMENTARY MATERIAL}

The Supplementary Material for this article can be found online at: https://www.frontiersin.org/articles/10.3389/fnana. 2019.00022/full\#supplementary-material

González Ballester, M. A., Zisserman, A. P., and Brady, M. (2002). Estimation of the partial volume effect in MRI. Med. Image Anal. 6, 389-405. doi: 10.1016/s1361-8415(02)00061-0

Gordon, E. M., Laumann, T. O., Gilmore, A. W., Newbold, D. J., Greene, D. J., Berg, J. J., et al. (2017). Precision functional mapping of individual human brains. Neuron 95, 791.e7-807.e7. doi: 10.1016/j.neuron.2017.07.011

Greicius, M. D., Supekar, K., Menon, V., and Dougherty, R. F. (2009). Restingstate functional connectivity reflects structural connectivity in the default mode network. Cereb. Cortex 19, 72-78. doi: 10.1093/cercor/bhn059

Hadjikhani, N., and Roland, P. E. (1998). Cross-modal transfer of information between the tactile and the visual representations in the human brain: a positron emission tomographic study. J. Neurosci. 18, 1072-1084. doi: 10.1523/JNEUROSCI.18-03-01072.1998

Hubbard, C. S., Khan, S. A., Xu, S., Cha, M., Masri, R., and Seminowicz, D. A. (2015). Behavioral, metabolic and functional brain changes in a rat model of 
chronic neuropathic pain: a longitudinal MRI study. Neuroimage 107, 333-344. doi: 10.1016/j.neuroimage.2014.12.024

Kalthoff, D., Po, C., Wiedermann, D., and Hoehn, M. (2013). Reliability and spatial specificity of rat brain sensorimotor functional connectivity networks are superior under sedation compared with general anesthesia. NMR Biomed. 26, 638-650. doi: 10.1002/nbm.2908

Lerner, A., Bagic, A., Hanakawa, T., Boudreau, E. A., Pagan, F., Mari, Z., et al. (2008). Involvement of insula and cingulate cortices in control and suppression of natural urges. Cereb. Cortex 19, 218-223. doi: 10.1093/cercor/ bhn074

LeVay, S., and Sherk, H. (1981). The visual claustrum of the cat. I. structure and connections. J. Neurosci. 1, 956-980. doi: 10.1523/JNEUROSCI.01-09-00 956.1981

Lipowska, M., Kowiański, P., Majak, K., Jagalska-Majewska, H., and Moryś, J. (2000). The connections of the endopiriform nucleus with the insular claustrum in the rat and rabbit. Folia Morphol. 59, 77-83. Available online at: https://journals.viamedica.pl/folia_morphologica/article/view/16534/13167

Mathur, B. N. (2014). The claustrum in review. Front. Syst. Neurosci. 8:48. doi: $10.3389 /$ fnsys. 2014.00048

Mathur, B. N., Caprioli, R. M., and Deutch, A. Y. (2009). Proteomic analysis illuminates a novel structural definition of the claustrum and insula. Cereb. Cortex 19, 2372-2379. doi: 10.1093/cercor/bhn253

McGeorge, A., and Faull, R. (1989). The organization of the projection from the cerebral cortex to the striatum in the rat. Neuroscience 29, 503-537. doi: 10.1016/0306-4522(89)90128-0

McIntosh, A. R. (2000). Towards a network theory of cognition. Neural Netw. 13, 861-870. doi: 10.1016/s0893-6080(00)00059-9

Muschelli, J., Nebel, M. B., Caffo, B. S., Barber, A. D., Pekar, J. J., and Mostofsky, S. H. (2014). Reduction of motion-related artifacts in resting state fMRI using aCompCor. Neuroimage 96, 22-35. doi: 10.1016/j.neuroimage. 2014.03.028

Naghavi, H. R., Eriksson, J., Larsson, A., and Nyberg, L. (2007). The claustrum/insula region integrates conceptually related sounds and pictures. Neurosci. Lett. 422, 77-80. doi: 10.1016/j.neulet.2007.06.009

Nasrallah, F. A., Tay, H. C., and Chuang, K. H. (2014). Detection of functional connectivity in the resting mouse brain. Neuroimage 86, 417-424. doi: 10.1016/j.neuroimage.2013.10.025

Olson, C., and Graybiel, A. (1980). Sensory maps in the claustrum of the cat. Nature 288, 479-481. doi: 10.1038/288479a0

Paasonen, J., Stenroos, P., Salo, R. A., Kiviniemi, V., and Gröhn, O. (2018). Functional connectivity under six anesthesia protocols and the awake condition in rat brain. Neuroimage 172, 9-20. doi: 10.1016/j.neuroimage.2018. 01.014

Patel, A. X., Kundu, P., Rubinov, M., Jones, P. S., Vértes, P. E., Ersche, K. D., et al. (2014). A wavelet method for modeling and despiking motion artifacts from resting-state fMRI time series. Neuroimage 95, 287-304. doi: 10.1016/j. neuroimage.2014.03.012

Power, J. D., Plitt, M., Laumann, T. O., and Martin, A. (2017). Sources and implications of whole-brain fMRI signals in humans. Neuroimage 146, 609-625. doi: 10.1016/j.neuroimage.2016.09.038

Qadir, H., Krimmel, S. R., Mu, C., Poulopoulos, A., Seminowicz, D. A., and Mathur, B. N. (2018). Structural connectivity of the anterior cingulate cortex, claustrum, and the anterior insula of the mouse. Front. Neuroanat. 12:100. doi: 10.3389/fnana.2018.00100

Remedios, R., Logothetis, N. K., and Kayser, C. (2010). Unimodal responses prevail within the multisensory claustrum. J. Neurosci. 30, 12902-12907. doi: 10.1523/JNEUROSCI.2937-10.2010
Reser, D. H., Majka, P., Snell, S., Chan, J. M., Watkins, K., Worthy, K., et al. (2017). Topography of claustrum and insula projections to medial prefrontal and anterior cingulate cortices of the common marmoset (callithrix jacchus). J. Comp. Neurol. 525, 1421-1441. doi: 10.1002/cne.24009

Segundo, J. P., and Machne, X. (1956). Unitary responses to afferent volleys in lenticular nucleus and claustrum. J. Neurophysiol. 19, 325-339. doi: 10.1152/jn. 1956.19.4.325

Smith, J. B., and Alloway, K. D. (2010). Functional specificity of claustrum connections in the rat: interhemispheric communication between specific parts of motor cortex. J. Neurosci. 30, 16832-16844. doi: 10.1523/JNEUROSCI.443810.2010

Sommers, M. G., van Egmond, J., Booij, L. H., and Heerschap, A. (2009). Isoflurane anesthesia is a valuable alternative for $\alpha$-chloralose anesthesia in the forepaw stimulation model in rats. NMR Biomed. 22, 414-418. doi: 10.1002/ nbm. 1351

Spector, I., Hassmannova, J., and Albe-Fessard, D. (1974). Sensory properties of single neurons of cat's claustrum. Brain Res. 66, 39-65. doi: 10.1016/00068993(74)90077-8

Voorn, P., Vanderschuren, L. J., Groenewegen, H. J., Robbins, T. W., and Pennartz, C. M. (2004). Putting a spin on the dorsal-ventral divide of the striatum. Trends Neurosci. 27, 468-474. doi: 10.1016/j.tins.2004.06.006

Wang, Q., Ng, L., Harris, J. A., Feng, D., Li, Y., Royall, J. J., et al. (2017). Organization of the connections between claustrum and cortex in the mouse. J. Comp. Neurol. 525, 1317-1346. doi: 10.1002/cne.24047

Watson, G. D., Smith, J. B., and Alloway, K. D. (2017). Interhemispheric connections between the infralimbic and entorhinal cortices: the endopiriform nucleus has limbic connections that parallel the sensory and motor connections of the claustrum. J. Comp. Neurol. 525, 1363-1380. doi: 10.1002/cne.23981

White, M. G., Cody, P. A., Bubser, M., Wang, H., Deutch, A. Y., and Mathur, B. N. (2017). Cortical hierarchy governs rat claustrocortical circuit organization. J. Comp. Neurol. 525, 1347-1362. doi: 10.1002/cne.23970

White, M. G., and Mathur, B. N. (2018). Frontal cortical control of posterior sensory and association cortices through the claustrum. Brain Struct. Funct. 223, 2999-3006. doi: 10.1007/s00429-018-1661-x

White, M. G., Panicker, M., Mu, C., Carter, A. M., Roberts, B. M., Dharmasri, P. A., et al. (2018). Anterior cingulate cortex input to the claustrum is required for top-down action control. Cell Rep. 22, 84-95. doi: 10.1016/j.celrep.2017. 12.023

Williams, K. A., Magnuson, M., Majeed, W., LaConte, S. M., Peltier, S. J., $\mathrm{Hu}$, X., et al. (2010). Comparison of $\alpha$-chloralose, medetomidine and isoflurane anesthesia for functional connectivity mapping in the rat. Magn. Reson. Imaging 28, 995-1003. doi: 10.1016/j.mri.2010.03.007

Woo, C., Krishnan, A., and Wager, T. D. (2014). Cluster-extent based thresholding in fMRI analyses: pitfalls and recommendations. Neuroimage 91, 412-419. doi: 10.1016/j.neuroimage.2013.12.058

Conflict of Interest Statement: The authors declare that the research was conducted in the absence of any commercial or financial relationships that could be construed as a potential conflict of interest.

Copyright (c) 2019 Krimmel, Qadir, Hesselgrave, White, Reser, Mathur and Seminowicz. This is an open-access article distributed under the terms of the Creative Commons Attribution License (CC BY). The use, distribution or reproduction in other forums is permitted, provided the original author(s) and the copyright owner(s) are credited and that the original publication in this journal is cited, in accordance with accepted academic practice. No use, distribution or reproduction is permitted which does not comply with these terms. 